\title{
Postoperative pancreatic fistulas after pancreaticoduodenectomy using the pair-watch suturing and duct-to-mucosa pancreaticojejunostomy anastomosis techniques
}

Nattapon Passawart ( $\square$ nattapon_sxbhu@hotmail.com )

Bhumibol Adulyadej Hospital

Warit Rungsrithananon

Bhumibol Adulyadej Hospital

Warakorn Jaseanchiun

Bhumibol Adulyadej Hospital

\section{Research Article}

Keywords: Pancreaticoduodenectomy, pancreatic fistula, sutures

Posted Date: February 9th, 2022

DOI: https://doi.org/10.21203/rs.3.rs-892593/v2

License: (a) This work is licensed under a Creative Commons Attribution 4.0 International License. Read Full License 


\section{Abstract}

Background/Aims: Morbidity and mortality after pancreaticoduodenectomy (PD) have been associated with postoperative pancreatic fistula (POPF). Pancreatic anastomosis is challenging for most surgeons, and there is no universal or standardized technique. This study compared the incidence of POPF between the pair-watch suturing technique (PWST) and duct-to-mucosa pancreaticojejunostomy (PJ) anastomosis technique.

Methods: This retrospective cohort analysis included 71 patients who underwent a PD between January 2009 and October 2018. The incidence and risk factors of complications after PWST and duct-to-mucosa PJ anastomoses were compared.

Results: There was no significant difference in the incidence of POPF between the PWST $(n=7 ; 30.4 \%)$ and duct-to-mucosa PJ ( $n=9 ; 18.7 \%)$ groups. The tumor site $(p=0.001)$ and pancreas density on computed tomography without contrast $(\mathrm{p}=0.002)$ were significantly different between the groups. Age $\geq 60$ years (odds ratio [OR]: 11.07; 95\% confidence interval [CI]: 1.14-107.36; $p=0.038$ ) and pancreatic body mass/duct size (B/W) ratio (OR: $1.41 ; 95 \% \mathrm{Cl}: 1.04-1.91 ; \mathrm{p}=0.029)$ were identified as significant risk factors for POPF. International Study Group for Pancreatic Fistula grade B POPF, wound infections, and pneumonia occurred more frequently in patients who underwent a duct-to-mucosa PJ anastomosis. However, the postoperative complications were not significantly different between the groups.

Conclusions: The incidence of clinically relevant POPF was similar between patients who underwent the PWST and those who underwent a duct-to-mucosa PJ anastomosis. However, a preoperative risk factor assessment for the evaluation of the tumor site, patient age, and B/W ratio could help determine which surgical technique should be used in individual patients.

\section{Introduction}

The standard surgery for patients with resectable and curable periampullary tumors that include the head of the pancreas, distal bile duct, duodenum, and ampulla is a standard pancreaticoduodenectomy (PD). PD is associated with high morbidity and mortality rates of approximately $30 \%-50 \%$ [1]. Postoperative pancreatic fistula (POPF) is a common postoperative complication occurring in $12.9 \%$ of patients undergoing PD and can lead to death [2]. Several techniques have been developed to reduce the incidence of POPF, though these techniques are complicated and distinctive [3]. The main site of POPF is believed to be the pancreatic anastomosis with the small intestine [4]. The two most-used techniques for the pancreatojejunostomy (PJ) anastomosis include the invagination anastomosis and the duct-to-mucosa anastomosis [5]. The reported incidence of POPF after duct-to-mucosa anastomosis (4.2\%) is lower than that after invagination anastomosis (26.4\%) [6]. The duct-to-mucosa anastomosis technique reduces postoperative pancreatic duct dilatation and atrophy more effectively than the invagination anastomosis 
technique. However, no standard technique for reducing the incidence of pancreatic leakage has been accepted [2].

A 2015 review of surgical anastomoses described the pair-watch suturing technique (PWST) that can be used to achieve a PJ anastomosis during PD [5]. The PWST is less complicated than other techniques and results in a secure anastomosis for pancreatic ducts of any size. Minimal severe complications have been reported after PD with the PWST. This retrospective cohort study aimed to compare the incidence of POPF between patients who underwent PD using the PWST and duct-to-mucosa anastomosis techniques.

\section{Patients And Methods}

\section{Patients}

A total of 71 patients who were diagnosed with periampullary tumors, including adenocarcinomas, endocrine tumors, and intraductal papillary mucinous neoplasms, or other tumors that required PD, at our hospital between January 2009 and October 2018 were enrolled in this study. All diagnoses were confirmed using helical computed tomography (CT) and endoscopic retrograde cholangiopancreatography. Patients with distant metastases, local unresectable lesions, or gastric involvement were excluded from the study. Prior to 2017, a duct-to-mucosa PJ anastomosis was used exclusively by multiple surgeons at our institution. After 2017, the PWST was performed by a single surgeon at our institution. Patients were divided into two groups according to the anastomosis technique used during PD: duct-to-mucosa PJ anastomosis (Group A, n=48) and PWST (Group B, n=23). Data regarding the patients' characteristics, preoperative data, and postoperative complications were retrospectively collected.

\section{Anastomosis types}

\section{Duct-to-mucosa PJ anastomosis}

A conventional duct-to-mucosa anastomosis was performed, as previously described [7]. Approximately 6-8 absorbable interrupted sutures (Novasorb ${ }^{\text {'tw }} 3-0$, Novamedic, Thailand) were placed between the posterior surface of the pancreas and the seromuscular layer of the jejunum. Subsequently, the jejunum was opened using an electrocautery device, and the pancreatic duct and jejunal mucosa opening were fixed with approximately 6-8 absorbable interrupted sutures (Novasorb ${ }^{\mathrm{m}} 4-0$, Novamedic) over the stent to form an end-to-side anastomosis. The anterior surfaces of the pancreas and jejunum were then approximated with absorbable interrupted sutures (Novasorb ${ }^{\text {tw }} 3-0$, Novamedic) in a process similar to that used on the posterior surface. 
The PWST was performed as previously described by Azumi et al. [5]. After sufficient pancreatectomy and mobilization of the pancreas, an end-to-side PJ was conducted as a two-layer anastomosis, including duct-to-mucosa and pancreatic parenchymal to jejunal seromuscular anastomoses. A surgical scope with a magnification of $1.5-2.5 x$ was used. A No. 5 over-feeding tube was used, and the opening side of the jejunal mucosa was regarded as the left side of the watch, while the pancreatic duct was regarded as the right side. The duct-to-mucosa anastomosis at the posterior side was performed first using absorbable interrupted sutures (PDS ${ }^{\text {tw }} 5-0$, Ethicon) from the nine o'clock position of the pancreatic site to the three o' clock position of the jejunal site using an outside sutured knot. Subsequently, the posterior surface of the pancreas was matched and sutured from the six o'clock position to the 12 o'clock position. The pairs remained hanging after suturing. Next, the pancreatic site was sutured on the anterior side from the one o'clock position to the five o'clock position, and each pair hung before being sutured to the jejunum side (Fig. 1). The pairs were then tied for approximation from the posterior side of the pancreas to the anterior side of the jejunum (Fig. 2). Subsequently, the pancreatic parenchyma and seromuscular of the jejunum were sutured with absorbable interrupted sutures (Novasorb ${ }^{\text {T"}} 4-0$, Novamedic).

\section{Definition of POPF}

POPF was defined as the presence of measurable output drainage fluid with an amylase level greater than three times the upper institutional normal serum amylase. Examinations to diagnose POPF were conducted on postoperative days 3,5 , and 7 . The severity of POPF was classified into clinically relevant grades (A, B, and C), according to the International Study Group on Pancreatic Fistula (ISGPF). This POPF grading system was based on parameters, such as clinical condition, treatment used, imaging study results, persistent drainage, re-operation, death, infection signs, and readmission [7].

\section{Statistical analysis}

All statistical analyses were conducted using SPSS software (version 27, IBM Corp., Armonk, NY, USA). Continuous data are presented as mean \pm standard deviation and categorical variables as frequency or percentage. The Mann-Whitney U-test was performed to compare continuous data with asymmetrical distribution, and independent t-tests were performed to compare continuous data with symmetrical distribution. The chi-square and Fisher's exact tests were performed to compare categorical variables. Univariate and multivariate analyses and binary logistic regression analyses were performed to analyze the risk factors associated with the development of POPF. Variables with $p$-values of $<0.2$ in univariate analysis were included in multivariate analysis. Statistical significance was set at a $p$-value of $<0.05$. 


\section{Results}

\section{Patient characteristics}

Overall, 48 patients who underwent a duct-to-mucosa PJ anastomosis and 23 patients who underwent the PWST for anastomosis were included in this study. The tumor site was significantly different between the groups ( $p=0.001$ ) with a higher proportion of patients in the duct-to-mucosa anastomosis group with tumors of the ampulla $(n=24 ; 53.5 \%)$ and in the PWST group with tumors of the pancreatic head $(n=15$; $68.2 \%$ ) (Table 1). The duct-to-mucosa anastomosis group had a significantly higher pancreatic density (38 \pm 7.4 Hounsfield units) than in the PWST group (35.8 \pm 15.6 Hounsfield units) on non-contrast CT $(p=0.002)$. The patients' age, sex, body mass index $(\mathrm{BMI})$, diagnoses, and preoperative and postoperative evaluations were not significantly different between the groups (Table 1).

\section{Incidence and risk factors of POPF}

The incidence of POPF was not significantly different between the groups (Table 2). Patients with POPF had a smaller main pancreatic duct (median: $2.7 \mathrm{~mm}$; range: $1.9-2.9 \mathrm{~mm}$ ) than those without POPF (median: $3.4 \mathrm{~mm}$; range: $2.3-6.1 \mathrm{~mm}$ ), though the difference was not significant. In the univariate analysis, tumor site, patient age, BMI, presence of diabetes mellitus, presence of cardiovascular disease, history of cholangitis, preoperative biliary drainage, serum albumin, main pancreatic duct size, the ratio of the body mass of the pancreas/pancreatic duct size (B/W ratio), operative time, estimated blood loss, and diagnosis were not identified as risk factors for POPF. In the multivariate logistic regression analysis, patients aged $\geq 60$ years (odds ratio [OR]: $11.07 ; 95 \%$ confidence interval [CI]: $1.14-107.36 ; p=0.038$ ) and higher $\mathrm{B} / \mathrm{W}$ ratio (OR: $1.41 ; 95 \% \mathrm{Cl}: 1.04-1.91 ; \mathrm{p}=0.029)$ were identified as significant risk factors for POPF.

\section{Postoperative complications}

More patients who underwent duct-to-mucosa PJ anastomoses had ISGPF grade B POPF, wound infections, and pneumonia than those who underwent PWST anastomoses, though there were no significant differences in postoperative complications between the groups (Table 3). No patient had pancreatitis, haemobilia, or ulcers.

\section{Discussion}

The incidence of POPF after duct-to-mucosa PJ anastomoses was similar to that after PWST anastomoses in this study. These results are consistent with those reported by Rajesh et al., who found no differences in the POPF rates among patients who underwent various PJ techniques $[8,9]$. These results suggest that the PWST or duct-to-mucosa PJ anastomoses can be performed successfully on the basis of the patient's condition. 
Another previous study reported similar POPF rates between duct-to-mucosa anastomoses and invagination anastomoses [10]. While several studies have reported that the invagination technique results in superior outcomes for patients requiring high-risk anastomoses [9,11], others reported that the invagination technique is not superior to duct-to-mucosa anastomoses for the reduction of POPF, although the rate of clinically relevant POPFs was reduced [12]. These results are consistent with the fact that invagination anastomosis is an easier technique during surgeries involving small pancreatic ducts [13]. However, studies have reported that the rate of clinically relevant POPF is lower in patients treated with duct-to-mucosa anastomoses [10]. Callery et al. reported that the reconstruction of the pancreatic remnant via PJ resulted in a lower incidence of POPF than the reconstruction via pancreaticogastrostomy and that the duct-to-mucosa anastomosis technique resulted in a lower rate of POPFs [14]. An important risk factor for POPF is the pancreatic duct size; in particular, a pancreatic duct size of $<3 \mathrm{~mm}$ is a risk factor for POPF [10]. These previous results are consistent with those of this study, in which patients in the POPF group had a smaller median pancreatic duct and a higher rate of POPF. In this study, patients who underwent duct-to-mucosa anastomoses had a lower rate of ISGPF grade A POPF ( $22.2 \%$ vs. $42.8 \%$, respectively) and a higher rate of ISGPF grade B POPF (66.7\% vs. $42.9 \%$, respectively) compared to patients who underwent PWST. The use of the PWST may reduce the severity and clinical relevance of POPF. As the selection of surgical techniques for high-risk anastomosis is dependent on several factors, the surgeon must decide which technique to use in each patient. The PWST is less complicated and provides reliable anastomoses for patients with a variety of pancreatic duct sizes [5]. Further studies comparing the invagination and PWST anastomosis techniques in small pancreatic ducts are required. The use of the appropriate anastomosis technique may reduce the incidence of POPF as POPF is a severe complication that is associated with specific surgical techniques [15]. The development of a standardized technique for PD may reduce the incidence of POPF [16], although no currently available technique has been proven to reduce the incidence of POPF after PD [17].

In this study, most patients undergoing PD prior to 2017 had ampullary tumors (53.5\%), while most patients undergoing PD after 2017 had pancreatic head tumors (68.2\%). A previous study reported that pancreatic head tumors had significantly decreased incidences of POPF, which may be due to the parameters associated with inflammation of the parenchyma or obstruction of the pancreatic duct leading to pancreatic firmness [18]. However, a soft pancreas has been associated with fatty infiltration. A small main pancreatic duct has also been associated with clinically relevant POPF after PD [19]. Although a higher rate of POPF was observed in the PWST group in this study due to a higher rate of pancreatic head tumors, the mean pancreatic density in the PWST group was less than that in the duct-to-mucosa anastomosis group. These results may be because pancreatic density on CT with lower attenuation implies higher fat accumulation [20], and a soft pancreas is a risk factor for POPF. In this study, patients aged $\geq 60$ years had a higher risk of POPF, which is consistent with the findings of previous studies $[21,22]$. Non-modifiable factors (age, BMI, and comorbidities), pathological factors (histotype, gland texture, and duct size), perioperative bleeding, and blood transfusions during surgery have been associated with POPF risk [15]. However, in this study, only patient age and B/W ratio were identified as risk factors for POPF. One study reported used preoperative CT to predict the development of POPFs 
according to duct size [23]. Another study reported that the largest single risk factor for POPF is narrowing of the pancreatic duct [24]. The B/W ratio can be used to predict the incidence of POPF [25]. These previous reports are consistent with the results of this study regarding $\mathrm{B} / \mathrm{W}$ ratio.

This study has some limitations, including its retrospective nature. In addition, the tumor sites were significantly different between the two groups, which may have led to a selection bias. However, this study also identified risk factors for POPF other than those previously reported. These results may be useful for the development of preoperative risk assessments, although surgeons may also refer to pancreatic pathology and other perioperative data regarding the tumor site prior to determining the appropriate surgical technique for each patient.

The incidence of POPF was not significantly different between patients who underwent duct-to-mucosa and PWST anastomoses. The tumor site, pancreatic density, and B/W ratio should be considered to choose suitable surgical techniques and reduce the incidence of POPF. Further prospective studies with larger populations are required to determine the best technique to achieve pancreatic anastomosis during PD.

\section{Declarations}

\section{ACKNOWLEDGMENTS}

The authors thank Prof. Mathirut Mungthin of Phramongkutklao College of Medicine for assisting with the design of this study.

\section{CONFLICT OF INTERESTS}

The authors declare that they have no conflict of interests.

\section{ETHICS APPROVAL AND CONSENT TO PARTICIPATE}

The protocol for this research project has been approved by a suitably constituted Ethics Committee of the institution (Approval No. 32/61), and it conforms to the provisions of the Declaration of Helsinki.

\section{CONSENT FOR PARTICIPATION}

The requirement of informed consent was waived due to the retrospective nature of the study. The study is registered on www.thaiclinicaltrials.org with the Unique Identifier Number TCTR20210721006. https://www.thaiclinicaltrials.org/show/TCTR20210721006

\section{CONSENT FOR PUBLICATION}

Not applicable.

\section{AVAILABILITY OF DATA AND MATERIALS}


The original anonymous dataset is available upon reasonable request from the corresponding author.

\section{AUTHORS' CONTRIBUTIONS}

NP offered the idea of this study and was a major contributor in writing the manuscript and collecting and analyzing data; WR collected patient data; and WJ was the supervisor of this study. All authors read and approved the final manuscript.

\section{References}

1. Kleespies A, Albertsmeier M, Obeidat F, Seeliger H, Jauch KW, Bruns CJ. The challenge of pancreatic anastomosis. Langenbecks Arch Surg 2008;393:459-471.

2. Schoellhammer HF, Fong Y, Gagandeep S. Techniques for prevention of pancreatic leak after pancreatectomy. Hepatobiliary Surg Nutr 2014;3:276-287.

3. Sun $\mathrm{H}, \mathrm{Ma} \mathrm{H}$, Hong G, Sun $\mathrm{H}$, Wang J. Survival improvement in patients with pancreatic cancer by decade: A period analysis of the SEER database, 1981-2010. Sci Rep 2014;4:6747.

4. Shimada K, Sano T, Sakamoto Y, Kosuge T. Clinical implications of combined portal vein resection as a palliative procedure in patients undergoing pancreaticoduodenectomy for pancreatic head carcinoma Ann Surg Oncol 2006;13:1569-1578.

5. Azumi Y, Isaji S, Kato H, Nobuoka Y, Kuriyama N, Kishiwada M, et al. A standardized technique for safe pancreaticojejunostomy: Pair-Watch suturing technique. World J Gastrointest Surg 2010;2:260-264.

6. Matsumoto Y, Fujii H, Miura K, Inoue S, Sekikawa T, Aoyama H, et al. Successful pancreatojejunal anastomosis for pancreatoduodenectomy. Surg Gynecol Obstet 1992;175:555-562.

7. Lee YN, Kim WY. Comparison of Blumgart versus conventional duct-to-mucosa anastomosis for pancreaticojejunostomy after pancreaticoduodenectomy. Ann Hepatobiliary Pancreat Surg 2018;22:253260.

8. Wang W, Zhang Z, Gu C, Liu Q, Liang Z, He W, et al. The optimal choice for pancreatic anastomosis after pancreaticoduodenectomy: A network meta-analysis of randomized control trials. Int J Surg 2018;57:111116.

9. Rajesh SS, Rajgopal A, Vikram AC, Manish SB, Shailesh VS. Pancreaticojejunostomy for pancreaticoenteric anastomosis after pancreaticoduodenectomy: one procedure with multiple techniques. Surg Pract Sci 2020;3:100019.

10. Bai X, Zhang Q, Gao S, Lou J, Li G, Zhang Y, et al. Duct-to-mucosa vs. invagination for pancreaticojejunostomy after pancreaticoduodenectomy: a prospective, randomized controlled trial from a single surgeon. J Am Coll Surg 2016;222:10-18. 
11. Ecker BL, McMillan MT, Asbun HJ, Ball CG, Bassi C, Beane JD, et al. Characterization and optimal management of high-risk pancreatic anastomoses during pancreatoduodenectomy. Ann Surg 2018;267:608-616.

12. Hua J, He Z, Qian D, Meng H, Zhou B, Song Z. Duct-to-mucosa versus invagination pancreaticojejunostomy following pancreaticoduodenectomy: a systematic review and meta-analysis. $J$ Gastrointest Surg 2015;19:1900-1909.

13. El Nakeeb A, El Hemaly M, Askr W, Ellatif MA, Hamed H, Elghawalby A, et al. Comparative study between duct to mucosa and invagination pancreaticojejunostomy after pancreaticoduodenectomy: a prospective randomized study. Int J Surg 2015;16:1-6.

14. Callery MP, Pratt, WB, Vollmer CM. Prevention and management of pancreatic fistula. J Gastrointest Surg 2009;13:163-173.

15. Søreide K, Labori KJ. Risk factors and preventive strategies for post-operative pancreatic fistula after pancreatic surgery: a comprehensive review. Scand J Gastroenterol 2016;51:1147-1154.

16. Shrikhande SV, Sivasanker M, Vollmer CM, Friess H, Besselink MG, Fingerhut A, et al. Pancreatic anastomosis after pancreatoduodenectomy: A position statement by the International Study Group of Pancreatic Surgery (ISGPS). Surgery 2017;161:1221-1234.

17. Kawaida H, Kono H, Hosomura N, Amemiya H, Itakura J, Fujii H, et al. Surgical techniques and postoperative management to prevent postoperative pancreatic fistula after pancreatic surgery. World $\mathrm{J}$ Gastroenterol 2019;25:3722-3737.

18. Williamsson C, Stenvall K, Wennerblom J, Andersson R, Andersson B, Tingstedt B. Predictive factors for postoperative pancreatic fistula-A Swedish nationwide register-based study. World J Surg 2020;44:4207-4213.

19. Sugimoto M, Takahashi S, Kojima M, Kobayashi T, Gotohda N, Konishi M. In patients with a soft pancreas, a thick parenchyma, a small duct, and fatty infiltration are significant risks for pancreatic fistula after pancreaticoduodenectomy. J Gastrointest Surg 2017;21:846-854.

20. Prachayakul V, Aswakul P. Pancreatic steatosis: what should gastroenterologists know? J Pancreas 2015;16:227-231.

21. Sukharamwala P, Thoens J, Szuchmacher M, Smith J, DeVito P. Advanced age is a risk factor for postoperative complications and mortality after a pancreaticoduodenectomy: a meta-analysis and systematic review. HPB (Oxford) 2012;14:649-657.

22. Casadei R, Ricci C, Lazzarini E, Taffurelli G, D’Ambra M, Mastroroberto M, et al. Pancreatic resection in patients 80 years or older: a meta-analysis and systematic review. Pancreas 2014;43:1208-1218. 
23. Roberts KJ, Storey R, Hodson J, Smith AM, Morris-Stiff G. Pre-operative prediction of pancreatic fistula: is it possible? Pancreatology 2013;13:423-428.

24. Roberts KJ, Karkhanis S, Pitchaimuthu M, Khan MS, Hodson J, Zia Z, et al. Comparison of preoperative CT-based imaging parameters to predict postoperative pancreatic fistula. Clin Radiol 2016;71:986-992.

25. Barbier L, Mège D, Reyre A, Moutardier VM, Ewald JA, Delpero JR. Predict pancreatic fistula after pancreaticoduodenectomy: ratio body thickness/main duct. ANZ J Surg 2018;88:E451-455.

\section{Tables}

Table 1. Patient characteristics 


\begin{tabular}{|c|c|c|c|c|}
\hline & $\begin{array}{l}\text { Total } \\
(n=71)\end{array}$ & $\begin{array}{l}\text { Duct-to- } \\
\text { mucosa } \\
\text { anastomosis } \\
(n=48)\end{array}$ & $\begin{array}{l}\text { Pair-watch } \\
\text { suturing } \\
\text { technique } \\
(n=23)\end{array}$ & $\mathbf{p}$ \\
\hline Age (years) & $60.9 \pm 12.0$ & $60.9 \pm 11.9$ & $61.0 \pm 12.7$ & $0.957^{c}$ \\
\hline Sex (male) & $45(63.4)$ & $33(68.8)$ & $12(52.2)$ & $0.175^{\mathrm{a}}$ \\
\hline BMI $\left(\mathrm{kg} / \mathrm{m}^{2}\right)$ & $22.6 \pm 4.0$ & $22.4 \pm 3.9$ & $23.0 \pm 4.2$ & $0.587^{\mathrm{c}}$ \\
\hline \multicolumn{5}{|l|}{ Diagnosis } \\
\hline Adenocarcinoma & $60(84.5)$ & $43(89.6)$ & $17(73.9)$ & $0.127^{b}$ \\
\hline Endocrine tumors & $1(1.4)$ & $0(0.0)$ & $1(4.4)$ & \\
\hline IPMN & $1(1.4)$ & $1(2.1)$ & $0(0.0)$ & \\
\hline Other & $9(12.7)$ & $4(8.3)$ & $5(21.7)$ & \\
\hline Biliary drainage & $35(49.3)$ & $22(45.8)$ & $13(56.5)$ & $0.399^{\mathrm{a}}$ \\
\hline History of cholangitis & $21(29.6)$ & $14(29.2)$ & $7(30.4)$ & $0.913^{\mathrm{a}}$ \\
\hline Hypertension & $34(47.9)$ & $22(45.8)$ & $12(52.2)$ & $0.617^{\mathrm{a}}$ \\
\hline Diabetes & $19(26.8)$ & $15(31.3)$ & $4(17.4)$ & $0.217^{\mathrm{a}}$ \\
\hline Cardiologic disease & $4(5.6)$ & $3(6.3)$ & $1(4.4)$ & $1.000^{\mathrm{b}}$ \\
\hline Smoking & $15(21.1)$ & $10(20.8)$ & $5(21.7)$ & $1.000^{\mathrm{b}}$ \\
\hline Alcohol consumption & $12(16.9)$ & $9(18.8)$ & $3(13.0)$ & $0.739^{b}$ \\
\hline History of abdominal operation & $7(9.9)$ & $4(8.3)$ & $3(13.0)$ & $0.674^{\mathrm{b}}$ \\
\hline Serum albumin (g/dL) & $3.7 \pm 0.6$ & $3.7 \pm 0.6$ & $3.8 \pm 0.6$ & $0.460^{\mathrm{C}}$ \\
\hline Serum $\mathrm{TB} \geq 3(\mathrm{mg} / \mathrm{dL})$ & $24(33.8)$ & $18(37.5)$ & $6(26.1)$ & $0.341^{\mathrm{a}}$ \\
\hline
\end{tabular}


$\mathrm{Hb}<10(\mathrm{~g} / \mathrm{dL})$

$8(11.3)$

$6(12.5)$

$2(8.7)$

$1.000^{\mathrm{b}}$

Operative time (min)

$432(365$, 505)
432 (354, 537)

EBL (ml)

\begin{tabular}{|c|c|}
\hline 700 (500, & 700 (500, \\
\hline 1500) & 1550) \\
\hline
\end{tabular}

$0.730^{\mathrm{d}}$

1500)

$65(94.2)$

$42(91.3)$

$23(100.0)$

$0.293^{\mathrm{a}}$

Pancreatic stent replacement $(n=69)$

\section{Tumor site $(n=67)$}

Ampulla

CBD

Duodenum

Periampullary

Pancreas head

Gall bladder

Blood transfusion (ml)

Length of stay (days)
$27(40.3)$

24 (53.3)

8 (11.9)

7 (15.6)

$2(4.4)$

$3(6.7)$

$8(17.8)$

$1(2.2)$

$1(1.5)$
$267(0,630)$

$23.1 \pm 20.1$
3 (13.6)

$1(4.6)$

$1(4.5)$

$2(9.1)$

$15(68.2)$

$0(0.0)$

$250(0,630) \quad 267(0,630) \quad 203.5(0,754) \quad 0.371^{d}$

$21.6 \pm 17.5 \quad 23.1 \pm 20.1$

$18.5 \pm 10.1$

$0.597^{\mathrm{d}}$

\section{Preoperative CT scan}

Pancreatic body thickness (B, mm)

$18.3 \pm 5.1$

$17.6 \pm 4.5$

$19.1 \pm 5.6$

$0.356^{\mathrm{c}}$

Main pancreatic duct size $(\mathrm{W}, \mathrm{mm})$

$2.9(2,5.4)$

$3.2(1.8,5.6)$

$2.8(2.3,3.9)$

$0.884^{\mathrm{d}}$

Body thickness/duct size ratio $5.8(3.3,8.7)$

$5.4(3.2,8.7)$

$6.6(4.3,8.6)$

(B/W ratio)

$$
37.1 \pm 11.5 \quad 38 \pm 7.4
$$

$35.8 \pm 15.6$

Pancreatic density

(without contrast (HU))

$88.8 \pm 16.2 \quad 90.1 \pm 13.8 \quad 87.1 \pm 19.2$

$0.592^{d}$

$0.002^{\mathrm{c}}$ 
(portal phase (HU))

Subcutaneous fat thickness (mm)
$14.3(8.9$, 19.8)
$13.1(8.6$,

18.8)
$16.8(12.3$ 20.8)

Data are presented as mean \pm standard deviation, median (range), or number (percentage).

Abbreviations: BMI, body mass index; IPMN, intraductal papillary mucinous neoplasm; TB, total bilirubin; Hb, hemoglobin; EBL; estimated blood loss; CBD, common bile duct; CT, computed tomography; HU, Hounsfield units.

${ }^{a}$ Chi-square test; ${ }^{b}$ Fisher's exact test; ${ }^{\mathrm{c}}$ Independent t-test, mean \pm standard deviation; ${ }^{\mathrm{d}}$ MannWhitney U-test, median (interquartile range)

Table 2. Factors associated with postoperative pancreatic fistulas 
Univariate

analysis

$\begin{array}{ll}\text { No POPF } & \text { POPF } \\ (\mathrm{n}=55) & (\mathrm{n}=16)\end{array}$

Odds ratio $\mathbf{P}$

(95\%

confidence

interval)
Multivariate

analysis

Adjusted

P

odds ratio

(95\%

confidence

interval)

\section{Tumor site}

CBD/Duodenum/GB $10(83.3)$

2 (16.7)

ref

Ampulla/Periampulla/

$43(78.2)$

$12(21.8)$

1.40

0.692

Pancreas head

(0.27-

7.25)

\section{Technique}

Pair-watch suturing

$16(69.6)$

7 (30.4)

ref

ref

technique

Duct-to-mucosa

39 (81.3)

$9(18.7)$

0.53

0.274

$1.14(0.23-$

0.873

anastomosis

(0.17-

5.60)

1.66)

\section{Age (years)}

\begin{tabular}{|c|c|c|c|c|c|c|}
\hline$<60$ & $25(86.2)$ & $4(13.8)$ & ref & & ref & \\
\hline \multirow[t]{3}{*}{$\geq 60$} & $30(71.4)$ & $12(28.6)$ & 2.50 & 0.151 & 11.07 & 0.038 \\
\hline & & & $(0.72-$ & & (1.14- & \\
\hline & & & $8.72)$ & & 107.36) & \\
\hline
\end{tabular}

$\mathrm{BMI} \geq 23\left(\mathrm{~kg} / \mathrm{m}^{2}\right)$

$23(69.7) \quad 10(30.3)$

2.32

0.150

(0.74-

7.29) 
Diabetes mellitus

$16(84.2)$

3 (15.8)

$0.56 \quad 0.415$

(0.14-

2.24)

Cardiovascular

$3(75.0)$

$1(25.0)$

1.16

0.903

disease

(0.11-

11.94)

History of cholangitis

\begin{tabular}{|c|c|c|c|c|c|c|}
\hline No & $40(80.0)$ & $10(20.0)$ & ref & & ref & \\
\hline Yes & $15(71.4)$ & $6(28.6)$ & $\begin{array}{c}1.6(0.49- \\
5.17)\end{array}$ & 0.432 & $\begin{array}{c}2.40(0.27- \\
21.28)\end{array}$ & 0.432 \\
\hline $\begin{array}{l}\text { Preoperative biliary } \\
\text { drainage }\end{array}$ & $28(80.0)$ & $7(20.0)$ & $\begin{array}{c}0.75 \\
(0.24- \\
2.30)\end{array}$ & 0.615 & & \\
\hline
\end{tabular}

\section{Serum Albumin}

$(g / d L)$

$<3$

$\geq 3$
$9(100.0) \quad 0(0.0)$

$46(74.2) \quad 16(25.8)$
N/A N/A

\section{Serum total bilirubin}

(mg/dL)

$\begin{array}{cccccc}<3(70.2) & 14(29.8) & 4.67 & 0.056 & 3.97(0.49- & 0.196 \\ & & (0.96- & 31.98) \\ & & 22.58) & \\ & & & & \\ & & & & \text { ref } & \text { ref }\end{array}$

Main pancreatic duct

$3.4(2.3$

$2.7(1.9,2.9)$

0.61

0.074

size $(\mathrm{mm})$

6.1)

(0.36-

1.05) 


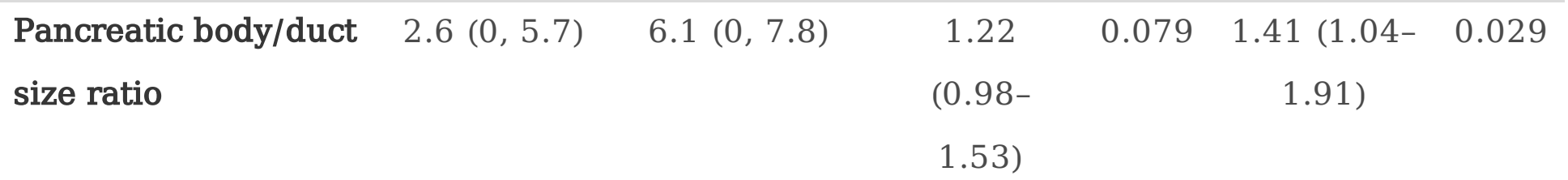

Operative time $\geq 360 \quad 42(76.4) \quad 13(23.6) \quad 1.34 \quad 0.681$

$\min \quad(0.33-$

$5.44)$

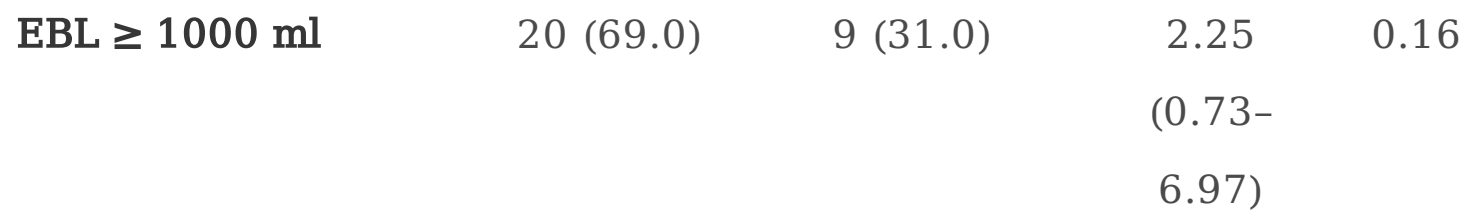

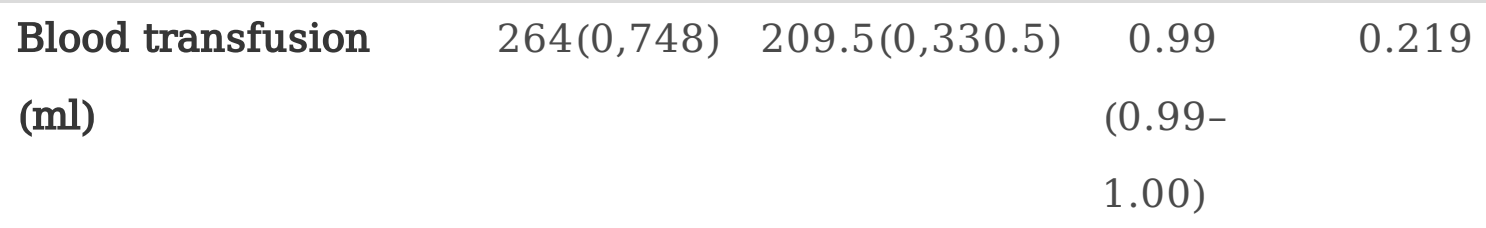

\section{Diagnosis}

Endocrine $\quad 6(54.5) \quad 5(45.5) \quad$ Ref

tumors/IPMN/other

$\begin{array}{lllll}\text { Adenocarcinoma } & 49(81.7) & 11(18.3) & 0.27 & 0.058\end{array}$

$(0.07-$

$1.04)$

Data are presented as median (range) or number (percentage).

Abbreviations: POPF, postoperative pancreatic fistula; CBD, common bile duct; GB, gall bladder; BMI, body mass index; EBL, estimate blood loss; IPMN, intraductal papillary mucinous neoplasm.

Goodness of fit test $=0.209$, Receiver operating characteristic curve $(\mathrm{ROC})=0.815$

Table 3. Postoperative complications 


\begin{tabular}{lcccc}
\hline Postoperative complication & Total & Duct-to-mucosa & Pair-watch suturing & \\
& $(\mathbf{n}=71)$ & anastomosis & technique & $\mathbf{p}$ \\
& & $(\mathbf{n}=48)$ & $(\mathrm{n}=23)$ & \\
\hline POPF & $16(22.5)$ & $9(18.7)$ & $7(30.4)$ & $0.270^{\mathrm{b}}$ \\
POPF-related death & $2(2.8)$ & $1(2.1)$ & $1(4.4)$ & $0.546^{\mathrm{b}}$ \\
\hline ISGPF grade of POPF & & & & \\
\hline A & $5(31.3)$ & $2(22.2)$ & $3(42.8)$ & $0.780^{\mathrm{b}}$ \\
\hline B & $9(56.3)$ & $6(66.7)$ & $3(42.9)$ & \\
\hline C & $2(12.5)$ & $1(11.1)$ & $1(14.3)$ & \\
\hline Wound infection & $16(22.54)$ & $13(27.1)$ & $3(13.0)$ & $0.185^{\mathrm{a}}$ \\
\hline Intra-abdominal abscess & $3(4.2)$ & $2(4.2)$ & $1(4.3)$ & $1.000^{\mathrm{b}}$ \\
\hline Cardiac event & $3(4.23)$ & $3(6.3)$ & $0(0.0)$ & $0.546^{\mathrm{b}}$ \\
\hline Bile leakage & $2(2.82)$ & $1(2.1)$ & $1(4.3)$ & $0.546^{\mathrm{b}}$ \\
\hline Pneumonia & $7(9.86)$ & $6(12.5)$ & $1(4.3)$ & $0.415^{\mathrm{b}}$ \\
\hline Lymphatic leakage & $2(2.82)$ & $0(0.00)$ & $2(8.7)$ & $0.102^{\mathrm{b}}$ \\
\hline & & & & \\
\hline
\end{tabular}

Data are presented as number (percentage).

Abbreviations: POPF, postoperative pancreatic fistula; ISGPF, International Study Group on Pancreatic Fistula.

${ }^{a}$ Chi-square test; ${ }^{b}$ Fisher's exact test

\section{Figures}

Figure 1 
Step-by-step pair-watch suturing technique.

After the posterior wall was sutured orderly from 1 to 7 , only the pancreatic site of the anterior wall was sutured from 8 to 12. Then the posterior wall sutures were tied, and the anterior wall was sutured to the jejunal site and tied.

\section{Figure 2}

Intraoperative photograph of pair-watch suturing technique.

a: An intraoperative image shows the pair-watch suturing technique (PWST) after 12 stitches have been sutured before the posterior knot is tied. Black arrow: Pancreatic remnant side. Asterisk: Jejunum side. b: The anastomosis is shown after the posterior surface of the duct-to-mucosa portion of the PWST has been approximated (green arrow). c: The anterior surface of the duct-to-mucosa portion of the PWST is approximated. The pancreatic parenchyma has not yet been sutured to the jejunal seromucosa. 